\title{
Unilateral Clearance for Primary Hyperparathyroidism in Selected Patients with Multiple Endocrine Neoplasia Type 1: Reply
}

\author{
Wouter P. Kluijfhout ${ }^{1} \cdot$ Quan-Yang Duh $^{1}$
}

Published online: 15 November 2016

(C) Société Internationale de Chirurgie 2016

We greatly appreciated the thoughtful comments and questions from our colleagues from the Sanjay Gandhi Postgraduate Institute of Medical Science regarding our recently published manuscript [1].

In our center, unilateral clearance (excising the abnormal gland, followed by excising the other gland on the same side as well as the ipsilateral cervical thymus) is only considered in patients with MEN1. In the absence of MEN1 syndrome, patients with single gland disease undergo minimally invasive parathyroidectomy, where only the abnormal gland is removed. This is a completely different surgical strategy. All patients who underwent unilateral clearance in our study had therefore a preoperative diagnosis of MEN1.

None of these eight patients who underwent unilateral clearance had more than 2 glands or supernumerary glands removed during their initial surgery. However, as noted in the manuscript, the one patient with recurrent disease had an ectopically located supernumerary gland in the tracheoesophageal groove on the same side as the initial surgery. This third gland in the same side was removed eight years later. It would likely have been missed even if the initial operation were bilateral exploration with subtotal resection.

Patients undergoing unilateral clearance were not only significantly younger, but also tended to have fewer symptoms from their disease. Three of eight patients were asymptomatic, versus two of 16 in the subtotal parathyroidectomy group. As suggested by Ahmad et al., we believe that these findings are the result of genetic heterogeneity. It is very likely that not all MEN1 patients have the same aggressive parathyroid disease, but rather that there is a spectrum of aggressiveness. This would also explain the higher rate of recurrent disease in the patients who underwent subtotal parathyroidectomy, despite their more aggressive initial surgery. Larger studies with longer follow-up will be necessary to investigate which mutations might be associated with less aggressive parathyroid disease. Until that time, imaging might help us to select patients who benefit from unilateral clearance with lower risk of permanent hypoparathyroidism.

\section{Reference}

1. Kluijfhout WP, Beninato T, Drake FT, Vriens MR, Gosnell J, Shen WT, Suh I, Liu C, Duh QY (2016) Unilateral clearance for primary hyperparathyroidism in selected patients with multiple endocrine neoplasia type 1. World J Surg 40:2964-2969
Wouter P. Kluijfhout

wpkluijfhout@gmail.com

1 Department of Surgery, University of California,

San Francisco - Mt Zion, 1600 Divisadero Street,

San Francisco, CA 94115, USA 